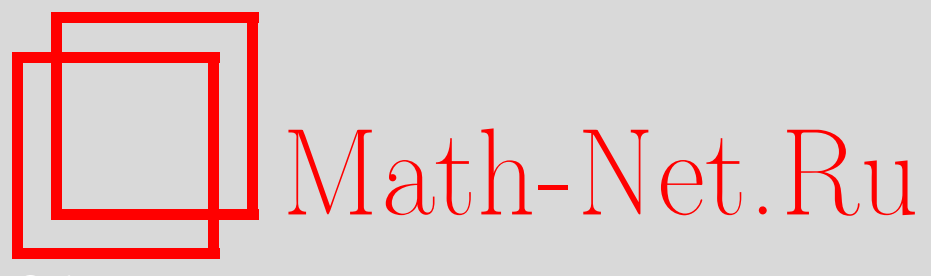

Э. Э. Боос, И. П. Волобуев, Ю. С. Михайлов, М. Н. Смоляков, Линеаризованная гравитация в модели РэндаллСундрума со стабилизированным расстоянием между бранами, ТМФ, 2006, том 149, номер 3, 339-353

DOI: https://doi.org/10.4213/tmf5521

Использование Общероссийского математического портала Math-Net.Ru подразумевает, что вы прочитали и согласны с пользовательским соглашением http://www . mathnet.ru/rus/agreement

Параметры загрузки:

IP : 18.209 .158 .208

26 апреля 2023 г., 18:21:43

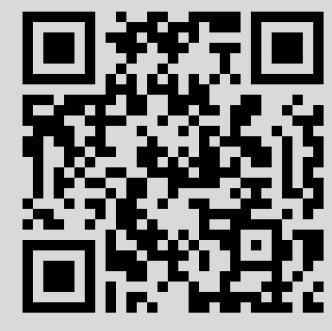




\title{
2006 г.
}

Э. Э. Боос*, И. П. Волобуев*, Ю. С. Михайлов ${ }^{\dagger}$, М. Н. Смоляков*

\section{ЛИНЕАРИЗОВАННАЯ ГРАВИТАЦИЯ В МОДЕЛИ РЭНДАЛЛ-СУНДРУМА СО СТАБИЛИЗИРОВАННЫМ РАССТОЯНИЕМ МЕЖДУ БРАНАМИ}

\begin{abstract}
Рассматривается линеаризованная гравитация в модели Рэндалл-Сундрума с двумя бранами, в которой стабилизация расстояния между бранами достигается путем введения скалярного поля Гольдбергера-Вайза. Построен лагранжиан второй вариации для флуктуаций гравитационного и скалярного полей над фоновым решением, изучена его калибровочная инвариантность, получены, расцеплены и решены соответствующие уравнения движения. Для физических степеней свободы этой модели получен эффективный четырехмерный лагранжиан, описывающий безмассовый гравитон, массивные гравитоны и набор массивных скалярных полей. Для этих полей также найдены массы и константы взаимодействия с материей на бране с отрицательным натяжением.
\end{abstract}

Ключевые слова: теории Калуцы-Клейна, браны, стабилизация радиона, линеаризованная гравитация.

\section{1. ВВЕДЕНИЕ}

В последнее время большое внимание уделяется моделям "мира на бране", в которых поля Стандартной модели предполагаются локализованными на трехмерном многообразии - мембране, или просто "бране", - вложенном в объемлющее многомерное пространство. В моделях мира на бране дополнительные измерения могут иметь большой или даже бесконечный размер и приводить к экспериментально наблюдаемым эффектам [1].

В работе [2] было найдено точное решение для системы двух бран, взаимодействующих с гравитацией в пятимерном пространстве-времени, которое позволяет учесть гравитационные поля, порождаемые бранами. Теория взаимодействий линеаризованной гравитации на фоне этого решения называется моделью Рэндалл-Сундрума,

*Научно-исследовательский институт ядерной физики им. Д. В. Скобельцына Московского государственного университета, Москва, Россия. E-mail: boos@theory.sinp.msu.ru, volobuev@theory.sinp.msu.ru, smolyakov@theory.sinp.msu.ru

${ }^{\dagger}$ Московский государственный университет, Москва, Россия. E-mail: mikhailov@theory.sinp.msu.ru 
и она широко обсуждается в литературе (см. [1]-[3] и библиографию в этих работах). Однако решение не фиксирует расстояние между бранами, т.е. оно может быть получено для произвольного значения этого расстояния. Поэтому небольшое изменение расстояния между бранами не меняет энергию системы, что проявляется в линеаризованной теории как появление безмассового скалярного поля - радиона. Взаимодействия этого поля оказываются противоречащими имеющимся экспериментальным данным. Таким образом, для того чтобы модель стала феноменологически приемлемой, необходимо стабилизировать расстояние между бранами, что эквивалентно появлению массы у радиона.

Известно, что этого можно достичь путем введения пятимерного скалярного поля $\phi$ с некоторыми потенциалом $V(\phi)$ во всем пространстве и дополнительными потенциалами $\lambda_{1,2}(\phi)$ на бранах [4]. Однако при таком подходе не учитывается влияние скалярного поля на фоновую метрику решения. Этот недостаток преодолен в работе [5], где найдены точные решения уравнений движения для всех полей. В результате стабилизация размера дополнительного измерения достигается благодаря граничным условиям для скалярного поля на бранах, а масса радиона определяется величиной отклонения фоновой метрики от решения Рэндалл-Сундрума.

Однако почти во всех работах по феноменологии модели Рэндалл-Сундрума попрежнему рассматривается нестабилизированнная модель, в которую масса радиона вводится "руками". В настоящей работе мы покажем, что такой подход представляется непоследовательным, так как появление массы у радиона приводит к изменению его константы связи с полями материи и значений всех параметров модели Рэндалл-Сундрума.

\section{2. ЛАГРАНЖИАН ВТОРОЙ ВАРИАЦИИ ДЛЯ СТАБИЛИЗИРОВАННОЙ МОДЕЛИ РЭНДАЛЛ-СУНДРУМА}

Рассмотрим в пятимерном пространстве-времени $E=M_{4} \times S^{1} / Z_{2}$ гравитацию, взаимодействующую с двумя мембранами и скалярным полем $\phi$. Обозначим координаты в $E$ как $\left\{\hat{x}^{M}\right\}=\left\{x^{\mu}, y\right\}, M=0,1,2,3,4$, где $\left\{x^{\mu}\right\}, \mu=0,1,2,3,-$ координаты в четырехмерном пространстве-времени, а координата $y \equiv x^{4}$ соответствует пятому измерению. Оно образует орбифолд $S^{1} / Z_{2}$, представляющий собой окружность диаметра $2 L / \pi$ с отождествленными точками $y$ и $-y$. В соответствии с этим метрика $g_{M N}$ и скалярное поле $\phi$ удовлетворяют условиям симметрии орбифолда

$$
\begin{aligned}
g_{\mu \nu}(x,-y) & =g_{\mu \nu}(x, y), & g_{\mu 4}(x,-y) & =-g_{\mu 4}(x, y), \\
g_{44}(x,-y) & =g_{44}(x, y), & \phi(x,-y) & =\phi(x, y) .
\end{aligned}
$$

Браны расположены в неподвижных точках орбифолда $y=0$ и $y=L$.

Действие этой модели записывается в виде

$$
S=S_{1}+S_{2},
$$

где гравитационное действие $S_{1}$ дается формулой

$$
S_{1}=\frac{1}{\hat{\kappa}^{2}} \int R \sqrt{-g} d^{5} \hat{x}
$$


а действие скалярного поля и мембран есть

$$
S_{2}=-\int\left(\frac{1}{2} \partial_{M} \phi \partial^{M} \phi+V(\phi)\right) \sqrt{-g} d^{5} \hat{x}-\int\left(\lambda_{1}(\phi) \delta(y)+\lambda_{2}(\phi) \delta(y-L)\right) \sqrt{-\tilde{g}} d^{5} \hat{x} .
$$

Здесь $\hat{\kappa}$ связана с пятимерной гравитационной постоянной $\widehat{G}$ соотношением $\hat{\kappa}=$ $\sqrt{16 \pi \widehat{G}}, \tilde{g}=\operatorname{det} \tilde{g}_{\mu \nu}, \tilde{g}_{\mu \nu}$ обозначает индуцированную на мембранах метрику. Сигнатура метрики $g_{M N}$ выбрана равной $(-,+,+,+,+)$. Индексы 1 и 2 различают браны. Интегрирование по $d y$ ведется по окружности $S^{1}$. Структуру потенциала скалярного поля $V(\phi)$ и потенциалов на бранах $\lambda_{1,2}(\phi)$ мы обсудим позже.

Решение для метрики будем искать в виде

$$
d s^{2}=\gamma_{M N} d x^{M} d x^{N}=e^{-2 A(y)} \eta_{\mu \nu} d x^{\mu} d x^{\nu}+d y^{2},
$$

сохраняющем пуанкаре-инвариантность в четырехмерном пространстве-времени. Здесь $\eta_{\mu \nu}$ есть метрика Минковского, а функции $A(y), \phi(y)$ определяются из уравнений движения

$$
\begin{gathered}
\frac{d V}{d \phi}+\frac{d \lambda_{1}}{d \phi} \delta(y)+\frac{d \lambda_{2}}{d \phi} \delta(y-L)=-4 A^{\prime} \phi^{\prime}+\phi^{\prime \prime} \\
\frac{6}{\hat{\kappa}^{2}}\left(A^{\prime}\right)^{2}+\frac{1}{2}\left(V-\frac{1}{2}\left(\phi^{\prime}\right)^{2}\right)=0 \\
\frac{1}{2}\left(\frac{1}{2}\left(\phi^{\prime}\right)^{2}+V+\lambda_{1} \delta(y)+\lambda_{2} \delta(y-L)\right)=-\frac{1}{\hat{\kappa}^{2}}\left(-3 A^{\prime \prime}+6\left(A^{\prime}\right)^{2}\right) .
\end{gathered}
$$

Для того чтобы найти аналитическое решение этой системы, мы воспользуемся результатами работ [5], [6]. Рассмотрим особый класс потенциалов, представимых в виде

$$
V(\phi)=\frac{1}{8}\left(\frac{d W}{d \phi}\right)^{2}-\frac{\hat{\kappa}^{2}}{12} W^{2}(\phi) .
$$

Нетрудно проверить, что если положить

$$
\phi^{\prime}(y)=\operatorname{sign}(y) \frac{1}{2} \frac{d W}{d \phi}, \quad A^{\prime}(y)=\operatorname{sign}(y) \frac{\hat{\kappa}^{2}}{12} W(\phi),
$$

где ${ }^{\prime} \equiv d / d y$, то уравнения движения справедливы всюду, за исключением бран. Для того чтобы уравнения выполнялись и на бранах, необходим согласованный выбор потенциала $V(\phi)$ и потенциалов на бранах $\lambda_{1,2}(\phi)$.

Выберем $W(\phi)$ в виде

$$
W=12 \frac{k}{\hat{\kappa}^{2}}-\phi^{2} u
$$

так что $V(\phi)$ представляет собой полином четвертой степени:

$$
V(\phi)=-\frac{\hat{\kappa}^{2} u^{2}}{12}\left(\phi^{2}-\frac{12 k+3 u}{\hat{\kappa}^{2} u}\right)^{2}+\frac{24 k u+3 u^{2}}{4 \hat{\kappa}^{2}} .
$$


Согласованные с ним потенциалы на бранах можно выбрать равными

$$
\begin{aligned}
& \lambda_{1}=W\left(\phi_{1}\right)+\frac{d W}{d \phi}\left(\phi_{1}\right)\left(\phi-\phi_{1}\right)+\beta_{1}^{2}\left(\phi-\phi_{1}\right)^{2}, \\
& \lambda_{2}=-W\left(\phi_{2}\right)-\frac{d W}{d \phi}\left(\phi_{2}\right)\left(\phi-\phi_{2}\right)+\beta_{2}^{2}\left(\phi-\phi_{2}\right)^{2} .
\end{aligned}
$$

Параметры потенциалов $k, u, \phi_{1,2}, \beta_{1,2}$, обезразмеренные фундаментальным пятимерным масштабом теории $M=\hat{\kappa}^{-2 / 3}$, следует считать положительными величинами порядка $O(1)$, т. е. в параметрах модели не должно содержаться иерархического различия масштабов.

Такой выбор потенциалов на бранах (10), (11) в рассматриваемом случае обеспечивает однозначность решения системы уравнений движения, которое в явном виде задается выражениями [5]

$$
\begin{gathered}
\phi(y)=\phi_{1} e^{-u|y|} \\
A(y)=k|y|+\frac{\hat{\kappa}^{2} \phi_{1}^{2}}{24} e^{-2 u|y|} .
\end{gathered}
$$

Данное решение является стабилизированным обобщением решения Рэндалл-Сундрума, что объясняет выбор суперпотенциала в виде (8). Расстояние между бранами связано с граничными условиями для поля $\phi$ и выражается через параметры потенциала соотношением

$$
L=\frac{1}{u} \ln \left(\frac{\phi_{1}}{\phi_{2}}\right) .
$$

Таким образом, мы видим, что расстояние между бранами, в отличие от рассматриваемого в модели Рэндалл-Сундрума без скалярного поля, стабилизировано. Отметим, что это достигается благодаря зависимости решения для скалярного поля от координаты дополнительного измерения. Вопрос единственности фонового решения (12), (13) при таком выборе потенциалов подробно обсуждается в работе [5].

Теперь построим лагранжиан второй вариации в модели Рэндалл-Сундрума со скалярным полем. Для этого параметризуем метрику и поле как

$$
g_{M N}=\gamma_{M N}+\hat{\kappa} h_{M N}, \quad \phi=\phi+\hat{\kappa} f,
$$

подставим в действие (2) и сохраним члены второго порядка по возмущениям. В результате получим

$$
\begin{aligned}
\frac{L_{g}}{\sqrt{-\gamma}=-} & \frac{1}{4}\left(\nabla_{S} h_{M N} \nabla^{S} h^{M N}+2 \nabla_{N} h \nabla_{M} h^{M N}-2 \nabla_{M} h^{M N} \nabla^{S} h_{S N}-\right. \\
& \left.-\nabla_{S} h \nabla^{S} h\right)+\left(A^{\prime}\right)^{2}\left[\frac{7}{2} h_{M N} h^{M N}-h h\right]- \\
& -A^{\prime \prime}\left[h_{M N} h^{M N}-\frac{1}{2} \tilde{h} h+\frac{1}{2} h_{M \nu} h^{M \nu}\right]+\frac{\hat{\kappa}^{2}}{2}\left[\frac{V}{2}\left(h_{M N} h^{M N}-\frac{1}{2} h h\right)+\right. \\
& +\frac{1}{2}\left(h_{\mu \nu} h^{\mu \nu}-\frac{1}{2} \tilde{h} \tilde{h}\right)\left[\lambda_{1} \delta(y)+\lambda_{2} \delta(y-L)\right]+
\end{aligned}
$$




$$
\begin{aligned}
& +\frac{1}{2}\left(\phi^{\prime}\right)^{2}\left(-\frac{1}{4} h h+\frac{1}{2} h_{M N} h^{M N}+h h_{44}-2 h_{4 M} h^{4 M}\right)- \\
& -f\left(h \frac{d V}{d \phi}+\tilde{h}\left[\frac{d \lambda_{1}}{d \phi} \delta(y)+\frac{d \lambda_{2}}{d \phi} \delta(y-L)\right]\right)-f^{\prime} \phi^{\prime} h+2 \partial_{M} f \phi^{\prime} h^{M 4}- \\
& \left.-\partial^{M} f \partial_{M} f-f^{2}\left(\frac{d^{2} V}{d \phi^{2}}+\frac{d^{2} \lambda_{1}}{d \phi^{2}} \delta(y)+\frac{d^{2} \lambda_{2}}{d \phi^{2}} \delta(y-L)\right)\right] .
\end{aligned}
$$

Здесь $h=\gamma_{M N} h^{M N}, \tilde{h}=\gamma_{\mu \nu} h^{\mu \nu}$, а $\nabla_{M}$ обозначает ковариантную производную в смысле метрики $\gamma_{M N}$.

Варьируя действие, соответствующее лагранжиану (15), и используя уравнения для фоновых полей, можно получить следующие уравнения движения для флуктуаций:

1) $\mu \nu$-компонента:

$$
\begin{aligned}
& \frac{1}{2}\left(\partial_{\sigma} \partial^{\sigma} h_{\mu \nu}-\partial_{\mu} \partial^{\sigma} h_{\sigma \nu}-\partial_{\nu} \partial^{\sigma} h_{\sigma \mu}+\partial_{4} \partial_{4} h_{\mu \nu}\right)+\frac{1}{2} \partial_{\mu} \partial_{\nu} \tilde{h}+\frac{1}{2} \partial_{\mu} \partial_{\nu} h_{44}- \\
& \quad-\frac{1}{2} \partial_{4}\left(\partial_{\mu} h_{4 \nu}+\partial_{\nu} h_{4 \mu}\right)+A^{\prime}\left(\partial_{\mu} h_{4 \nu}+\partial_{\nu} h_{4 \mu}\right)+\frac{1}{2} \gamma_{\mu \nu}\left(-\partial_{4} \partial_{4} \tilde{h}-\right. \\
& \quad-\partial_{\sigma} \partial^{\sigma} h_{44}-\partial_{\sigma} \partial^{\sigma} \tilde{h}+4 A^{\prime} \partial_{4} \tilde{h}-3 A^{\prime} \partial_{4} h_{44}+\partial_{\sigma} \partial_{\tau} h^{\sigma \tau}+2 \partial^{\sigma} \partial_{4} h_{\sigma 4}- \\
& \left.\quad-4 A^{\prime} \partial^{\sigma} h_{4 \sigma}\right)-h_{\mu \nu}\left(2 A^{\prime 2}-A^{\prime \prime}\right)+\frac{3}{2} h_{44} \gamma_{\mu \nu}\left(4 A^{\prime 2}-A^{\prime \prime}\right)- \\
& -\frac{\hat{\kappa}^{2}}{2}\left(\gamma_{\mu \nu} f^{\prime} \phi^{\prime}+f \gamma_{\mu \nu}\left(-4 A^{\prime} \phi^{\prime}+\phi^{\prime \prime}\right)\right)=0
\end{aligned}
$$

2) $\mu 4$-компонента:

$$
\partial_{4}\left(\partial_{\mu} \tilde{h}-\partial^{\nu} h_{\mu \nu}\right)+\partial^{\nu}\left(\partial_{\nu} h_{\mu 4}-\partial_{\mu} h_{\nu 4}\right)+3 A^{\prime} \partial_{\mu} h_{44}+\hat{\kappa}^{2} \partial_{\mu} f \phi^{\prime}=0
$$

3) 44-компонента:

$$
\partial^{\mu}\left(\partial^{\nu} h_{\mu \nu}-\partial_{\mu} \tilde{h}\right)-6 A^{\prime} \partial^{\mu} h_{\mu 4}+3 A^{\prime} \partial_{4} \tilde{h}+\hat{\kappa}^{2}\left(-h_{44} V-f \frac{d V}{d \phi}+f^{\prime} \phi^{\prime}\right)=0 ;
$$

4) уравнение для поля $f$ :

$$
\begin{aligned}
& h_{44}\left(\frac{d \lambda_{1}}{d \phi} \delta(y)+\frac{d \lambda_{2}}{d \phi} \delta(y-L)\right)-2 h_{44}\left(-4 A^{\prime} \phi^{\prime}+\phi^{\prime \prime}\right)+ \\
& +\phi^{\prime} \partial_{4} \tilde{h}-\phi^{\prime} \partial_{4} h_{44}-8 A^{\prime} \partial_{4} f-2 \phi^{\prime} \partial^{\mu} h_{\mu 4}+2 \partial_{M} \partial^{M} f- \\
& -2 f\left(\frac{d^{2} V}{d \phi^{2}}+\frac{d^{2} \lambda_{1}}{d \phi^{2}} \delta(y)+\frac{d^{2} \lambda_{2}}{d \phi^{2}} \delta(y-L)\right)=0,
\end{aligned}
$$

где $\partial_{4} \equiv \partial / \partial y$. Мы будем также использовать следующее вспомогательное уравнение, получающееся сверткой индексов в $\mu \nu$-уравнении:

$$
\begin{gathered}
\partial^{\mu} \partial^{\nu} h_{\mu \nu}-\partial^{\mu} \partial_{\mu}\left(\tilde{h}+\frac{3}{2} h_{44}\right)-6 A^{\prime} \partial_{4}\left(h_{44}-\tilde{h}\right)-\frac{3}{2} \partial_{4} \partial_{4} \tilde{h}-6 A^{\prime} \partial^{\mu} h_{\mu 4}+ \\
+3 \partial^{\mu} \partial_{4} h_{\mu 4}+6 h_{44}\left(4 A^{\prime 2}-A^{\prime \prime}\right)-2 \hat{\kappa}^{2}\left(f^{\prime} \phi^{\prime}+f\left(-4 A^{\prime} \phi^{\prime}+\phi^{\prime \prime}\right)\right)=0 .
\end{gathered}
$$




\section{3. ВЫБОР КАЛИБРОВКИ}

Обсудим теперь калибровочную инвариантность лагранжиана (15). Нетрудно проверить, что отвечающее этому лагранжиану действие инвариантно относительно калибровочных преобразований

$$
\begin{aligned}
h_{M N}^{(\prime)}(\hat{x}) & =h_{M N}(\hat{x})-\left(\nabla_{M} \xi_{N}+\nabla_{N} \xi_{M}\right), \\
f^{(\prime)}(\hat{x}) & =f(\hat{x})-\partial_{4} \phi \xi_{4},
\end{aligned}
$$

если функции $\xi^{M}(\hat{x})$ удовлетворяют условиям симметрии орбифолда

$$
\xi^{\mu}(x,-y)=\xi^{\mu}(x, y) \quad \xi^{4}(x,-y)=-\xi^{4}(x, y)
$$

(здесь и в нижеследующих формулах для калибровочных преобразований значок ( $/$ ) обозначает преобразованное поле).

Для метрики рассматриваемого типа эти калибровочные преобразования могут быть найдены явно; они имеют вид

$$
\begin{gathered}
h_{\mu \nu}^{(\prime)}(x, y)=h_{\mu \nu}(x, y)-\left(\partial_{\mu} \xi_{\nu}+\partial_{\nu} \xi_{\mu}-2 \gamma_{\mu \nu} \partial_{4} A \xi_{4}\right), \\
h_{\mu 4}^{(\prime)}(x, y)=h_{\mu 4}(x, y)-\left(\partial_{\mu} \xi_{4}+\partial_{4} \xi_{\mu}+2 \partial_{4} A \xi_{\mu}\right), \\
h_{44}^{(\prime)}(x, y)=h_{44}(x, y)-2 \partial_{4} \xi_{4}, \\
f^{(\prime)}(x, y)=f(x, y)-\partial_{4} \phi \xi_{4} .
\end{gathered}
$$

Аналогичные калибровочные преобразования для тензорного поля в модели Рэндалл-Сундрума без скалярного поля обсуждались в работах [7]-[9]. Мы будем использовать их для выделения физических степеней свободы полей $h_{M N}$ и $f$.

Найдем калибровку, которая позволит упростить уравнения движения. Для этого покажем, что на поля можно наложить условие

$$
\left(e^{-2 A} h_{44}\right)^{\prime}-\frac{2}{3} \hat{\kappa}^{2} e^{-2 A} \phi^{\prime} f=0 .
$$

Отметим, что в отсутствие скалярного поля, т.е. когда действие теории переходит в действие оригинальной модели Рэндалл-Сундрума [2], эта калибровка соответствует волновой функции радиона в дополнительном измерении, описанной в работе [9] (см. также [10]).

Из формул (23) следует, что уравнение для функции $\xi_{4}$, переводящей произвольную полевую конфигурацию в конфигурацию, удовлетворяющую условию (24), имеет вид

$$
\partial_{4} \partial_{4} \xi_{4}-2 A^{\prime} \partial_{4} \xi_{4}-\frac{1}{3} \hat{\kappa}^{2}\left(\phi^{\prime}\right)^{2} \xi_{4}=-\frac{1}{2}\left(\partial_{4} h_{44}-2 A^{\prime} h_{44}-\frac{2}{3} \hat{\kappa}^{2} \phi^{\prime} f\right) .
$$

Стандартная теория решения дифференциальных уравнений предполагает непрерывность коэффициента перед $\partial_{4} \xi_{4}$. Однако в нашем случае он имеет разрыв. Для устранения этого недостатка перепишем уравнение в следующем виде:

$$
\begin{aligned}
\partial_{4} \partial_{4}\left(\xi_{4} e^{-A}\right)+\xi_{4} e^{-A}\left(A^{\prime \prime}-\left(A^{\prime}\right)^{2}-\frac{1}{3} \hat{\kappa}^{2}\left(\phi^{\prime}\right)^{2}\right)= \\
=-\frac{1}{2} e^{-A}\left(\partial_{4} h_{44}-2 A^{\prime} h_{44}-\frac{2}{3} \hat{\kappa}^{2} \phi^{\prime} f\right) \equiv \omega(x, y) .
\end{aligned}
$$


Функции $\left(A^{\prime}\right)^{2}$ и $\left(\phi^{\prime}\right)^{2}$ являются гладкими функциями $y$. Хотя функция $A^{\prime \prime}$ имеет сингулярности в точках $y=0, y=L$, сингулярные члены выпадают из уравнения вследствие того, что $\xi_{4}$ обращается в этих точках в нуль. Таким образом, множитель перед $\xi_{4} e^{-A}$ можно считать гладкой функцией, и это уравнение можно решать стандартными методами относительно функции $\xi_{4} e^{-A}$ на отрезке $[0, L]$.

Однородное уравнение, соответствующее $(25)$, на отрезке $[0, L]$ имеет два независимых решения, которые мы обозначаем $\chi_{1}$ и $\chi_{2}$. Для нахождения $\xi_{4}$ воспользуемся методом функций Грина. В результате имеем

$$
\begin{aligned}
\xi_{4}(x, y) & =e^{A} \int_{0}^{L} \frac{-\chi_{1}(y) \chi_{2}(z)+\chi_{2}(y) \chi_{1}(z)}{\chi_{1}^{\prime}(z) \chi_{2}(z)-\chi_{2}^{\prime}(z) \chi_{1}(z)} \theta(y-z) \omega(x, z) d z- \\
& -e^{A} \frac{\chi_{1}(y) \chi_{2}(0)-\chi_{2}(y) \chi_{1}(0)}{\chi_{1}(L) \chi_{2}(0)-\chi_{2}(L) \chi_{1}(0)} \int_{0}^{L} \frac{-\chi_{1}(L) \chi_{2}(z)+\chi_{2}(L) \chi_{1}(z)}{\chi_{1}^{\prime}(z) \chi_{2}(z)-\chi_{2}^{\prime}(z) \chi_{1}(z)} \omega(x, z) d z .
\end{aligned}
$$

Нетрудно проверить, что найденная функция $\xi_{4}$ обращается в нуль на концах отрезка и вследствие этого может быть продолжена до нечетной функции на интервале $(-L, L)$.

Для фонового решения (12), (13) функции $\chi_{1}$ и $\chi_{2}$ могут быть найдены явно:

$$
\begin{gathered}
\chi_{1}(y)=e^{-\varsigma / 2} \varsigma^{s} F(-n, 2 s+1, \varsigma), \\
\chi_{2}(y)=e^{-\varsigma / 2} \varsigma^{-s} F(-n-2 s,-2 s+1, \varsigma), \\
\varsigma=\frac{\sqrt{\beta}}{u} e^{-2 y}, \quad n=\frac{\alpha}{4 \sqrt{\beta} u}-s-\frac{1}{2}, \\
\beta=\frac{\hat{\kappa}^{4} u^{2}}{12^{2}}, \quad \alpha=\frac{\hat{\kappa}^{2} u(k-u)}{6}, \quad s=\frac{k}{2 u},
\end{gathered}
$$

где $F$ - гипергеометрическая функция. Таким образом, для выбранного нами фонового решения калибровка (24) действительно существует. Отметим еще, что соотношение (24) было получено ранее (см. работу [11]) как уравнение настоящей работы (17), в котором сохранены только скалярные степени свободы. Точно так же, как в нестабилизированной модели Рэндалл-Сундрума, выбором калибровки можно обратить в нуль поле $h_{\mu 4}[7]$; при этом уравнение (17) становится уравнением связи.

После этого остаются еще калибровочные преобразования, удовлетворяющие условию

$$
\partial_{4}\left(e^{2 A} \xi_{\mu}\right)=0
$$

Итак, в рассматриваемой модели существует калибровка

$$
\left(e^{-2 A} h_{44}\right)^{\prime}-\frac{2}{3} \hat{\kappa}^{2} e^{-2 A} \phi^{\prime} f=0, \quad h_{\mu 4}=0 .
$$

Для явного выделения физических степеней свободы данной модели представим гравитационное поле в виде

$$
h_{\mu \nu}=b_{\mu \nu}+\frac{1}{4} \gamma_{\mu \nu} \tilde{h}
$$


где $b_{\mu \nu}$ - бесследовое тензорное поле $\left(\gamma^{\mu \nu} b_{\mu \nu}=0\right)$. В дальнейшем мы увидим, что поле $b_{\mu \nu}(x, y)$ описывает безмассовый гравитон и массивные тензорные поля КалуцыКлейна. Подставляя калибровочные условия (30) и подстановку (31) в $\mu 4$-уравнение и свернутое $\mu \nu$-уравнение (20), получим

$$
\begin{gathered}
-\partial_{4}\left(\partial^{\nu} b_{\mu \nu}\right)+\frac{3}{4} \partial_{\mu} \partial_{4}\left(\tilde{h}+2 h_{44}\right)=0 \\
\partial^{\mu} \partial^{\nu} b_{\mu \nu}-\frac{3}{4} \partial_{\rho} \partial^{\rho} \tilde{h}-\frac{3}{2} \partial_{\rho} \partial^{\rho} h_{44}-\frac{3}{2} \frac{\partial^{2}}{\partial y^{2}} \tilde{h}+6 A^{\prime} \partial_{4} \tilde{h}-3 \frac{\partial^{2}}{\partial y^{2}} h_{44}+12 A^{\prime} \partial_{4} h_{44}=0 .
\end{gathered}
$$

Вид первого из этих уравнений подсказывает подстановку $\tilde{h}=-2 h_{44}$, которая позволяет расцепить уравнения для полей $b_{\mu \nu}, h_{44}$ и $f$. Действительно, в результате такой подстановки предыдущие уравнения принимают вид

$$
\begin{aligned}
\partial_{4}\left(\partial^{\nu} b_{\mu \nu}\right) & =0, \\
\partial^{\mu} \partial^{\nu} b_{\mu \nu} & =0 .
\end{aligned}
$$

Рассматривая законы преобразования поля $b_{\mu \nu}$ и его дивергенции, можно показать, что остаточные калибровочные преобразования (29) позволяют наложить калибровку $\partial^{\nu} b_{\mu \nu}=0[7]$.

Таким образом, существует калибровка

$$
\begin{aligned}
\left(e^{-2 A} h_{44}\right)^{\prime}-\frac{2}{3} \hat{\kappa}^{2} e^{-2 A} \phi^{\prime} f & =0, \\
h_{\mu 4} & =0, \\
\tilde{b}=\gamma_{\mu \nu} b^{\mu \nu}=0, & \partial^{\nu} b_{\mu \nu}=0 .
\end{aligned}
$$

После наложения этой калибровки остаются калибровочные преобразования, удовлетворяющие условиям

$$
\xi_{\mu}=e^{-2 A} \epsilon_{\mu}(x), \quad \partial^{\nu} \epsilon_{\nu}(x)=0, \quad \square \epsilon_{\nu}=0,
$$

которые обеспечивают правильное число физических степеней свободы у безмассового гравитона.

\section{4. УРАВНЕНИЯ ДВИЖЕНИЯ}

Вернемся к рассмотрению уравнений движения. Очевидно, что в калибровке (35) после подстановки $\tilde{h}=-2 h_{44}$ свернутое $\mu \nu$-уравнение $(20)$ и $\mu 4$-уравнение выполняются тождественно. Уравнение для $\mu \nu$-компонент сводится к уравнению для поперечно-бесследового поля $b_{\mu \nu}$ :

$$
\frac{1}{2}\left(\partial_{\sigma} \partial^{\sigma} b_{\mu \nu}+\frac{\partial^{2}}{\partial y^{2}} b_{\mu \nu}\right)-b_{\mu \nu}\left(2\left(A^{\prime}\right)^{2}-A^{\prime \prime}\right)=0 .
$$

Отметим, что в это уравнение не входит фоновое скалярное поле $\phi$, и оно совершенно аналогично соответствующему уравнению в нестабилизированной модели. 
Для того чтобы найти уравнения движения для $h_{44}$, нужно разрешить калибровочное условие (35) относительно функции $f$ и подставить последнюю в уравнения (18), (19). Это можно сделать, либо вводя естественную регуляризацию $(\operatorname{sign}(y))^{2}=1$, либо ограничивая уравнения на интервал $(0, L)$ и учитывая присутствующие в них сингулярные члены с помощью граничных условий. Последний метод оказывается технически более простым, и мы будем использовать его.

Уравнение для 44-компоненты (18) заметно упрощается, если его переписать на интервале $(0, L)$, вводя новую функцию $g e^{-2 A(y)} h_{44}(x, y)$, а также используя связь потенциала $V$ с $A$ и $\phi(6)$ :

$$
g^{\prime \prime}+2 g^{\prime}\left(A^{\prime}-\frac{\phi^{\prime \prime}}{\phi^{\prime}}\right)-\frac{\hat{\kappa}^{2}\left(\phi^{\prime}\right)^{2}}{3} g+\partial_{\mu} \partial^{\mu} g=0 .
$$

Отметим, что в терминах $g$ подстановка (31) и калибровочное условие (35), pacцепляющие уравнения движения, принимают вид

$$
\begin{gathered}
h_{\mu \nu}=b_{\mu \nu}-\frac{1}{2} \eta_{\mu \nu} g, \quad h_{44}=e^{2 A(y)} g, \\
g^{\prime}-\frac{2}{3} \hat{\kappa}^{2} e^{-2 A} \phi^{\prime} f=0, \\
h_{\mu 4}=0, \quad \tilde{b}=\gamma_{\mu \nu} b^{\mu \nu}=0, \quad \partial^{\nu} b_{\mu \nu}=0 .
\end{gathered}
$$

Калибровочное условие (40), разрешенное относительно $f$ на интервале $(0, L)$, перепишется как

$$
f=\frac{3}{2 \hat{\kappa}^{2}} \frac{e^{2 A}}{\phi^{\prime}} g^{\prime} .
$$

Подстановка этого выражения в уравнение (19) приводит к уравнению, получающемуся дифференцированием уравнения (38) по $y$, и граничным условиям на бранах:

$$
\begin{aligned}
\left(\frac{1}{2} \frac{d^{2} \lambda_{1}}{d \phi^{2}}-\frac{\phi^{\prime \prime}}{\phi^{\prime}}\right) g^{\prime}+\left.\partial_{\mu} \partial^{\mu} g\right|_{y=+0}=0, \\
\left(\frac{1}{2} \frac{d^{2} \lambda_{2}}{d \phi^{2}}+\frac{\phi^{\prime \prime}}{\phi^{\prime}}\right) g^{\prime}-\left.\partial_{\mu} \partial^{\mu} g\right|_{y=L-0}=0 .
\end{aligned}
$$

Итак, наш выбор калибровки и соответствующая подстановка позволили расцепить уравнения движения. Таким образом, флуктуации метрики и скалярного поля на фоне обобщенного решения Рэндалл-Сундрума описываются поперечнобесследовым тензорным полем $b_{\mu \nu}$ и скалярным полем $g$. Их классические уравнения движения даются формулами (37) и (38), (41), соответственно.

Рассмотрим сначала моды тензорного поля $b_{\mu \nu}$, которое удовлетворяет уравнению (37). Прежде всего отметим, что точным решением для нулевой моды будет

$$
\psi_{0}(y)=N_{0} e^{-2 A}
$$

Для других мод найти точные решения в общем случае не удается. Поэтому далее мы будем использовать приближение $u L \ll 1$ (в дальнейшем окажется, что следует 
взять $u L \sim 0.1)$, которое представляется достаточно общим и физически интересным [12].

Сохраняя в $A$ только члены, линейные по $y$, найдем

$$
A(y)=\tilde{k}|y|, \quad \tilde{k}=k-\frac{\hat{\kappa}^{2} \phi_{1}^{2}}{12} u .
$$

Таким образом, в этом приближении уравнение (37) для тензорного поля фактически совпадает с уравнением нестабилизированной модели при замене $k \rightarrow \tilde{k}$, и его можно решить аналитически. Соответствующие формулы для собственных значений и функций нулевых и ненулевых мод достаточно подробно рассмотрены в работе [7]. В частности, в этом приближении нормированные функции $\psi_{0}$, отвечающие нулевой массе $m_{0}=0$, в координатах, галилеевых на бране в $y=0$, имеют вид

$$
\psi_{0}(y)=N_{0} e^{-2 \tilde{k}|y|}, \quad N_{0}=\frac{\tilde{k}^{1 / 2}}{\left(1-e^{-2 \tilde{k} L}\right)^{1 / 2}},
$$

а в координатах, галилеевых на бране в $y=L,-$

$$
\psi_{0}(y)=N_{0} e^{-2 \tilde{k}|y|+2 \tilde{k} L}, \quad N_{0}=\frac{\tilde{k}^{1 / 2}}{\left(e^{2 \tilde{k} L}-1\right)^{1 / 2}}
$$

(напомним, что координаты называются галилеевыми, если $\left.g_{\mu \nu}=(-1,1,1,1)[13]\right)$. Представляя $b_{\mu \nu}$ в виде

$$
b_{\mu \nu}=\sum_{n=0}^{\infty} b_{\mu \nu}^{n}(x) \psi_{n}(y)
$$

подставляя это выражение в (15), опуская скалярные поля и интегрируя по $y$, можно получить эффективный лагранжиан для тензорного поля, точно совпадающий с полученным в работе [7] при замене $k \rightarrow \tilde{k}$.

Рассмотрим теперь уравнение (38) для скалярного поля с граничными условиями (41). Для того чтобы найти спектр масс скалярных частиц, описываемых уравнением (38), будем искать $g$ в виде

$$
g(x, y)=e^{i p x} g_{n}(y), \quad p^{2}=-\mu_{n}^{2} .
$$

В результате уравнение и граничные условия для $g_{n}(y)$ примут вид

$$
\begin{gathered}
g_{n}^{\prime \prime}+2 A^{\prime} g_{n}^{\prime}-2 \frac{\phi^{\prime \prime}}{\phi^{\prime}} g_{n}^{\prime}-\frac{\hat{\kappa}^{2}\left(\phi^{\prime}\right)^{2}}{3} g_{n}=-\mu_{n}^{2} e^{2 A} g_{n}, \\
\left(\beta_{1}^{2}+u\right) g_{n}^{\prime}+\left.\mu_{n}^{2} e^{2 A} g_{n}\right|_{y=+0}=0, \\
\left(\beta_{2}^{2}-u\right) g_{n}^{\prime}-\left.\mu_{n}^{2} e^{2 A} g_{n}\right|_{y=L-0}=0,
\end{gathered}
$$

где в граничных условиях учтен явный вид функций $\phi(y)$ и $\lambda_{1,2}$.

Поскольку в граничные условия (48), (49) входят собственные значения, оператор в уравнении (47) оказывается несамосопряженным. Однако уравнение (47) и граничные условия (48), (49) можно объединить в одно уравнение на окружности $S^{1}$ :

$$
\left(\frac{e^{2 A}}{\left(\phi^{\prime}\right)^{2}} g_{n}^{\prime}\right)^{\prime}-\frac{\hat{\kappa}^{2} e^{2 A}}{3} g_{n}=-\mu_{n}^{2} \frac{e^{4 A}}{\left(\phi^{\prime}\right)^{2}}\left(1+\frac{2}{\beta_{1}^{2}+u} \delta(y)+\frac{2}{\beta_{2}^{2}-u} \delta(y-L)\right) g_{n} .
$$


Это уравнение записано в форме Штурма-Лиувилля, и нетрудно понять, что оператор в задаче для собственных значений для этого уравнения на окружности $S^{1}$ самосопряженный (задача с периодическими граничными условиями). Единственная необычная черта этого уравнения - то, что весовая функция является обобщенной. Из общей теории [14] следует, что если $\beta_{2}^{2}>u$, то все собственные значения рассматриваемой задачи действительны и положительны. Собственные функции этой задачи $\left\{g_{n}(y)\right\}$ образуют полную систему и могут быть выбраны действительными. Собственные функции, отвечающие разным собственным значениям, ортогональны с обобщенным весом, который определяется уравнением (50). Поэтому их можно нормировать условием

$$
\frac{9 \mu_{n}^{2}}{4 \hat{\kappa}^{2}}\left(\int_{-L}^{L} d y \frac{e^{4 A}}{\left(\phi^{\prime}\right)^{2}} g_{n} g_{k}+\left.\frac{2}{\left(\beta_{1}^{2}+u\right)} \frac{e^{4 A}}{\left(\phi^{\prime}\right)^{2}} g_{n} g_{k}\right|_{y=0}+\left.\frac{2}{\left(\beta_{2}^{2}-u\right)} \frac{e^{4 A}}{\left(\phi^{\prime}\right)^{2}} g_{n} g_{k}\right|_{y=L}\right)=\delta_{n k}
$$

Пятимерное скалярное поле $g(x, y)$ можно разложить по этой системе:

$$
g(x, y)=\sum_{n=1}^{\infty} \varphi_{n}(x) g_{n}(y)
$$

где четырехмерные скалярные поля $\varphi_{n}(x)$ имеют массы $\mu_{n}^{2}$.

Для нахождения собственных функций и собственных значений в явном виде удобнее использовать не уравнение (50), а уравнение (47) с граничными условиями (48), (49), при этом мы также будем использовать приближение $u L \ll 1$, которое использовалось при решении уравнения для тензорного поля. Тогда имеем

$$
u y=u L \frac{y}{L}<u L \ll 1 .
$$

Учитывая явный вид $A$ и $\phi$, уравнение (47) можно записать как

$$
g_{n}^{\prime \prime}+2 A^{\prime} g_{n}^{\prime}+2 u g^{\prime}-\frac{\hat{\kappa}^{2} \phi_{1}^{2}}{3} u^{2} g_{n}+\mu_{n}^{2} e^{2 A} g_{n}=0 .
$$

Будем решать это уравнение в координатах, галилеевых на бране в $L$. В этом случае $A(y)$ выражается через $\tilde{k}(43)$ в виде

$$
A(y)=\tilde{k}(y-L) .
$$

Как мы уже установили, все собственные значения рассматриваемой задачи больше нуля. Поэтому введем новую переменную соотношением

$$
z=\frac{\mu_{n}}{\tilde{k}} e^{\tilde{k} y-\tilde{k} L}, \quad \frac{\mu_{n}}{\tilde{k}} e^{-\tilde{k} L} \leqslant z \leqslant \frac{\mu_{n}}{\tilde{k}} .
$$

В этой переменной уравнение перепишется как

$$
\frac{d^{2} g_{n}}{d z^{2}}+\left(3+\frac{2 u}{\tilde{k}}\right) \frac{1}{z} \frac{d g_{n}}{d z}+\left(1-\frac{b^{2}}{z^{2}}\right) g_{n}=0, \quad b^{2}=\frac{\hat{\kappa}^{2} \phi_{1}^{2}}{3} \frac{u^{2}}{\tilde{k}^{2}} .
$$


Будем искать $g_{n}$ в виде $g_{n}(z)=z^{a} t_{n}(z)$. Для $t_{n}$ получим уравнение

$$
\frac{d^{2} t_{n}}{d z^{2}}+\left(2 a+3+\frac{2 u}{\tilde{k}}\right) \frac{1}{z} \frac{d t_{n}}{d z}+\frac{a(a+2+2 u / \tilde{k})}{z^{2}} t_{n}+\left(1-\frac{b^{2}}{z^{2}}\right) t_{n}=0 .
$$

Для того чтобы привести это уравнение к виду уравнения Бесселя, нужно положить $a=-(1+u / \tilde{k})$. В результате получим

$$
\frac{d^{2} t_{n}}{d z^{2}}+\frac{1}{z} \frac{d t_{n}}{d z}+\left(1-\frac{\alpha^{2}}{z^{2}}\right) t_{n}=0, \quad \alpha^{2}=a^{2}+b^{2} .
$$

Общее решение этого уравнения -

$$
t_{n}(z)=A J_{\alpha}(z)+B J_{-\alpha}(z), \quad \alpha=\sqrt{a^{2}+b^{2}},
$$

причем функция $J_{\alpha}(z)$ регулярна в нуле, а функция $J_{-\alpha}(z)$ сингулярна. Соответственно

$$
g_{n}(z)=z^{-(1+u / \tilde{k})}\left(A J_{\alpha}(z)+B J_{-\alpha}(z)\right) .
$$

Граничные условия в терминах $z$ перепишутся в виде

$$
\begin{aligned}
\tilde{k} z^{2} g_{n}+\left.\left(\beta_{1}^{2}+u\right) z \frac{d g_{n}}{d z}\right|_{z_{1}=\left(\mu_{n} / \tilde{k}\right) e^{-\tilde{k} L}} & =0, \\
\tilde{k} z^{2} g_{n}-\left.\left(\beta_{2}^{2}-u\right) z \frac{d g_{n}}{d z}\right|_{z_{2}=\left(\mu_{n} / \tilde{k}\right)} & =0 .
\end{aligned}
$$

В следующем разделе мы покажем, что для воспроизведения ньютоновской гравитации на бране в $L$ при сильной пятимерной гравитации нужно взять $\tilde{k} L \sim 35$. В этом случае с большой точностью можно считать $z_{1}=\left(\mu_{n} / \tilde{k}\right) e^{-\tilde{k} L} \approx 0$, и граничное условие в нуле позволяет опустить сингулярный член в $g_{n}(z)$, так как $B / A \sim e^{-2 \tilde{k} L}$ и возникающие поправки пренебрежимо малы.

Итак, $g_{n}(z)$ с точностью до нормировки можно представить в виде

$$
g_{n}(z)=z^{-(1+u / \tilde{k})} J_{\alpha}(z) .
$$

Второе граничное условие при $z_{2}=\mu_{n} / \tilde{k}$ дает уравнение для спектра масс скалярных частиц:

$$
\left(1+\alpha+\frac{u}{\tilde{k}}+\frac{\tilde{k} z_{2}^{2}}{\beta_{2}^{2}-u}\right) J_{\alpha}\left(z_{2}\right)-z_{2} J_{\alpha-1}\left(z_{2}\right)=0 .
$$

Разлагая функции Бесселя при малых $z_{2}$ до второго члена и удерживая в уравнении члены порядка $z_{2}^{2}$, для массы низшего возбуждения получим

$$
\frac{\mu_{1}^{2}}{\tilde{k}^{2}}=\frac{4(-1+\alpha-u / \tilde{k})(1+\alpha)\left(\beta_{2}^{2}-u\right)}{4 \tilde{k}(1+\alpha)+(1+\alpha-u / \tilde{k})\left(\beta_{2}^{2}-u\right)} .
$$

В приближении малых $u / \tilde{k}$ это сводится к

$$
\mu_{1}^{2}=\frac{2}{3} \hat{\kappa}^{2} \phi_{1}^{2} u^{2} \frac{\beta_{2}^{2}-u}{\beta_{2}^{2}+4 \tilde{k}}
$$


что при $\beta_{2}^{2} \rightarrow \infty$ совпадает с результатами работ [11], [12] в рассматриваемом приближении. Следующие корни уравнения (59) имеют порядок $\tilde{k}^{2}$, а асимптотическая формула общей теории [14] для больших $n$ дает $\mu_{n}^{2}=\pi^{2} \tilde{k}^{2} n^{2}$.

Нормированные функции $g_{n}(y)$ имеют вид

$$
\begin{gathered}
g_{n}(y)=A_{n}\left(\frac{\mu_{n}}{\tilde{k}} e^{\tilde{k} y-\tilde{k} L}\right)^{-(1+u / \tilde{k})} J_{\alpha}\left(\frac{\mu_{n}}{\tilde{k}} e^{\tilde{k} y-\tilde{k} L}\right), \\
A_{n}=\frac{\sqrt{2} u \hat{\kappa} \phi_{1}\left(\mu_{n} / \tilde{k}\right)^{1+u / \tilde{k}}}{3 \mu_{n}} \times \\
\times\left[\frac{\sqrt{\pi}\left(\mu_{n} / \tilde{k}\right)^{\alpha-1} H\left(-\alpha, 2 \mu_{n} / \tilde{k}\right) \Gamma(-\alpha+3 / 2)}{2^{2(\alpha+1)} \tilde{k} \Gamma(2+\alpha) \Gamma(1+\alpha)}+\frac{J_{\alpha}^{2}\left(\mu_{n} / \tilde{k}\right)}{\beta_{2}^{2}-u}\right]^{-1 / 2} .
\end{gathered}
$$

Здесь $H(a, y)$ - функция Струве. В условии нормировки также опущены граничные члены в $y=0$, которыми можно пренебречь в используемом нами приближении.

Для того чтобы найти эффективное четырехмерное действие теории, подставим (31) и (39), (40) в лагранжиан (15) и, используя разложения (46), (52), учитывая нормировку (51) и интегрируя по координате дополнительного измерения, получим

$$
S_{\text {eff }}=-\frac{1}{4} \sum_{k=0}^{\infty} \int d x\left(\partial^{\sigma} b^{k, \mu \nu} \partial_{\sigma} b_{\mu \nu}^{k}+m_{k}^{2} b^{k, \mu \nu} b_{\mu \nu}^{k}\right)-\frac{1}{2} \sum_{k=1}^{\infty} \int d x\left(\partial_{\nu} \varphi_{k} \partial^{\nu} \varphi_{k}+\mu_{k}^{2} \varphi_{k} \varphi_{k}\right) .
$$

Таким образом, мы получили эффективный лагранжиан теории, представляющий собой сумму стандартных четырехмерных лагранжианов тензорных и скалярных полей.

\section{5. ГРАВИТАЦИОННОЕ ВЗАИМОДЕЙСТВИЕ НА БРАНАХ}

Взаимодействие четырехмерных полей $b_{\mu \nu}^{n}(x)$ и $\varphi_{n}(x)$ с полями на бранах определяется взаимодействием флуктуаций пятимерного гравитационного поля $h_{\mu \nu} \mathrm{c}$ материей на бранах, которое имеет вид

$$
\frac{\hat{\kappa}}{2} \int_{B_{1}} h_{\mu \nu}(x, 0) T_{\mu \nu}^{(1)} \sqrt{-\operatorname{det} \gamma_{\mu \nu}(0)} d x+\frac{\hat{\kappa}}{2} \int_{B_{2}} h_{\mu \nu}(x, L) T_{\mu \nu}^{(2)} \sqrt{-\operatorname{det} \gamma_{\mu \nu}(L)} d x,
$$

где $T_{\mu \nu}^{(1)}$ и $T_{\mu \nu}^{(2)}$ - тензоры энергии-импульса материи на бранах 1 и 2 , соответственно.

В дальнейшем мы будем рассматривать поля только на бране в $y=L$, которая считается "нашей". Подставляя в формулу (65) разложения (46) и (52), получим, что взаимодействие тензорных и скалярных полей с материей на бране в $y=L$ в галилеевых координатах имеет вид

$$
\frac{\hat{\kappa}}{2} \int_{B_{2}}\left(\psi_{0}(L) b_{\mu \nu}^{0}(x) T^{\mu \nu}+\sum_{n=1}^{\infty} \psi_{n}(L) b_{\mu \nu}^{n}(x) T^{\mu \nu}-\frac{1}{2} \sum_{n=1}^{\infty} g_{n}(L) \varphi_{n}(x) T_{\mu}^{\mu}\right) d x .
$$

Коэффициент перед нулевой модой тензорного поля $b_{\mu \nu}^{0}(x)$ выражается через четырехмерную гравитационную постоянную $G_{2}$ на бране в $y=L$. С учетом (45) 
получаем

$$
\frac{\sqrt{16 \pi G_{2}}}{2}=\frac{\hat{\kappa}}{2} N_{0}=\frac{\sqrt{16 \pi \widehat{G}}}{2} \frac{\tilde{k}^{1 / 2}}{\left(e^{2 \tilde{k} L}-1\right)^{1 / 2}} .
$$

Таким образом, четырехмерная гравитационная постоянная $G_{2}$ на бране 2 выражается через пятимерную константу $\widehat{G}$ следующим образом:

$$
G_{2}=\widehat{G} \frac{\tilde{k}}{e^{2 \tilde{k} L}-1} .
$$

Переписывая эту формулу в терминах массы Планка на бране с отрицательным натяжением и фундаментальной пятимерной массой $M$, получим

$$
M_{\mathrm{Pl}}^{2}=M^{3} \frac{e^{2 \tilde{k} L}-1}{\tilde{k}} .
$$

Опять в рассматриваемом приближении мы получили формулы, совпадающие с формулами для нестабилизированной модели при замене $k \rightarrow \tilde{k}$. Поэтому в данном случае, для того чтобы получить правильное значение гравитационной постоянной на бране с отрицательным натяжением, можно взять те же значения параметров, которые используются в нестабилизированной модели, т.е. $M \sim \tilde{k} \sim 1 \mathrm{~T}$ ЭВ, $\tilde{k} L \sim 35$ [7]. Тогда параметр $и$ может быть порядка десятков ГэВ, а масса радиона (61) - порядка сотен ГэВ.

Используя точное решение для нулевой моды (42), можно получить соотношения, связывающие массу Планка на бране с отрицательным натяжением и фундаментальную пятимерную массу, и вне рамок используемого в настоящей работе приближения. Другие возможные приближения рассмотрены в работе [12].

Как видно из выражения (66), константа взаимодействия $n$-й моды скалярного поля с материей определяется значением волновой функции моды на бране и задается выражением

$$
\epsilon_{n}=-\frac{\hat{\kappa} A_{n}}{4} J_{\alpha}\left(\frac{\mu_{n}}{\tilde{k}}\right)\left(\frac{\mu_{n}}{\tilde{k}}\right)^{-(1+u / \tilde{k})} .
$$

Для радиона эта константа взаимодействия принимает вид

$$
\epsilon_{1} \simeq-\frac{\mu_{1}}{\sqrt{24 M^{3} \tilde{k}}} \sqrt{\frac{\beta_{2}^{2}+4 \tilde{k}^{2}}{\beta_{2}^{2}-u}}
$$

и при определенных выше значениях параметров оказывается порядка $\epsilon_{1}^{-1} \sim 50$ ТэВ.

\section{6. ЗАКЛЮЧЕНИЕ}

В настоящей работе мы рассмотрели стабилизированную модель Рэндалл-Сундрума, построили для нее лагранжиан второй вариации и получили уравнения движения для полей, описывающих возмущения над фоновым решением. Были найдены удобные калибровка и подстановка, позволившие расцепить уравнения движения и выделить физические степени свободы модели. Оказалось, что сектор тензорных 
полей отщепляется от скалярного сектора и имеет ту же структуру, что и тензорный сектор нестабилизированной модели. При определенных значениях параметров стабилизированной модели влияние скалярного поля на тензорные возбуждения сводится к перенормировке параметра $k$ нестабилизированной модели, который заменяется на $\tilde{k}(43)$. В результате формулы для масс тензорных возбуждений и для связи четырехмерных и пятимерных гравитационных постоянных получаются из соответствующих формул нестабилизированной модели заменой $k \rightarrow \tilde{k}$.

Анализ скалярного сектора показал, что при определенном выборе параметров модели масса радиона может быть порядка сотен ГэВ, а обратный размер дополнительного измерения и массы тензорных полей - порядка 1 ТэВ. При этом константа связи радиона с материей на бране получилась примерно на порядок меньше, чем в нестабилизированной модели при тех же значениях параметров.

Необходимо еще отметить, что явный вид фоновых полей $A(y)$ и $\phi(y)$ мы использовали только для получения спектра масс и констант связи. Все результаты, связанные с выбором калибровки, расцеплением уравнений движения и структурой скалярного сектора, справедливы в любой модели, стабилизированной с помощью скалярного поля [15].

Благодарности. Авторы выражают благодарность С. Ю. Вернову, Ю. В. Грацу, П. Ф. Ермолову, Ю. А. Кубышину и В. А. Рубакову за полезные обсуждения. Работа была поддержана РФФИ (грант № 04-02-17448) и Программой поддержки ведущих научных школ (грант № НШ-8122.2006.2). Работа М.Н. Смолякова была также поддержана грантом MK-8718.2006.2 Президента Российской Федерации.

\section{Список литературы}

[1] В. А. Рубаков, УФН, 171 (2001), 913.

[2] L. Randall, R. Sundrum, Phys. Rev. Lett., 83 (1999), 3370.

[3] I. Antoniadis, Eur. Phys. J. C, 33 (2004), S914.

[4] W. D. Goldberger, M. B. Wise, Phys. Rev. Lett., 83 (1999), 4922.

[5] O. DeWolfe, D. Z. Freedman, S. S. Gubser, A. Karch, Phys. Rev. D, 62:4 (2000), 046008.

[6] A. Brandhuber, K. Sfetsos, JHEP, 10 (1999), 013.

[7] Э. Э. Боос, И. П. Волобуев, Ю. А. Кубышин, М. Н. Смоляков, ТМФ, 131:2 (2002), 216.

[8] I. Ya. Aref'eva, M. G. Ivanov, W. Muck, K. S. Viswanathan, I. V. Volovich, Nucl. Phys. B, 590 (2000), 273.

[9] Ch. Charmousis, R. Gregory, V. Rubakov, Phys. Rev. D, 62 (2000), 067505.

[10] M. Toharia, Mod. Phys. Lett. A, 19 (2004), 37.

[11] C. Csaki, M. L. Graesser, G. D. Kribs, Phys. Rev. D, 63 (2001), 065002.

[12] E. E. Boos, Yu. S. Mikhailov, M. N. Smolyakov, I. P. Volobuev, Nucl. Phys. B, 717 (2005), 19.

[13] Л.Д. Ландау, Е. М. Лифшиц, Теория поля, Наука, М., 1967.

[14] В. М. Бабич, М.Б. Капилевич, С. Г. Михлин и др., Линейные уравнения математической физики, Наука, М., 1964.

[15] E. E. Boos, Yu. S. Mikhailov, M. N. Smolyakov, I. P. Volobuev, Mod. Phys. Lett. A, 21 (2006), 1431; hep-th/0511185.

Поступила в редакцию 20.02.2006, после доработки 2.06.2006

2 Теоретическая и математическая физика, т. 149, № 3, 2006 г. 